(C) 1983. The Genetical Society of Great Britain

\title{
SEX CHROMOSOME EVOLUTION IN THE POLYTYPIC SPECIES PYCNOGASTER CUCULLATA
}

\author{
J. FERNANDEZ-PIQUERAS, A. RODRIGUEZ CAMPOS, C. SENTIS CASTAÑO \\ AND E. ROJO GARCIA \\ Departamento de Genética, Facultad de Ciencias, Universidad Autónoma \\ de Madrid, Mód. C-XV. Cantoblanco, Madrid-34. Spain
}

Received 29.x.82

\begin{abstract}
Pycnogaster cucullata (Charp.) is a polytypic species of Tettigonioidea having both $\mathrm{X} 0$ and neo $\mathrm{XY}$ populations. Moreover the neo $\mathrm{XY}$ populations are themselves of two distinct types referred to as neo $X Y_{1}$ and neo $X Y_{2}$.

A comparison between the $\mathrm{X} 0$ state and the two different neo $\mathrm{XY}$ systems, using C-banding and silver staining techniques, has served to clarify the differentiation of the two neo XY systems. In both cases, a centric fusion between the progenitor $\mathrm{M}_{2}$ chromosome, the second largest autosome, and the $\mathrm{X}$ of the $\mathrm{X} 0$ form has been accompanied by loss of an active NOR in the neo $\mathrm{Y}$, which is the direct derivative of the $M_{2}$. In both categories of neo XY sex bivalents, a secondary construction appears in the distal region of the $X_{L}$ limb, which gives a silver reaction similar to that observed at the centromeres. The neo $\mathrm{XY}_{2}$ system is distinctive in two respects in comparison with the neo $X Y_{1}$. In the first place it has an additional C-positive block located distally in the neo $Y$. Additionally there is also a novel C-block in the proximal half of the $X_{R}$ limb of the neo $X$. The neo $X Y_{2}$ system thus provides the first unambiguous example of the "secondary heterochromatinization" of a sex chromosome system within a single species.
\end{abstract}

\section{INTRODUCTION}

To-date it has been assumed that the differentiation of those chromosomes that carry the sex determining genes must have resulted from a restriction of recombination between two originally homologous chromosomes. In theory this can be achieved either by genes which specifically suppress crossing-over, by chiasma localisation, or by a structural rearrangement in, or a heterochromatinization of, the sex bivalent. In a recent paper, for example, Grossman et al. (1981) indicated that differentiation of $\mathrm{Z}$ and $\mathrm{W}$ chromosomes in the American schistosomes (Trematoda) resulted from translocation of part of one homologous sex chromosome on to another with subsequent heterochromatinization of the $\mathrm{W}$. In vertebrates with female heterogamety it has been concluded that the primary step in the differentiation of the $\mathrm{W}$ and the $\mathrm{Z}$ is the heterochromatinization of the $\mathrm{W}$ chromosome (Ray-Chaudhury et al., 1971; Schmid et al., 1979; Singh et al., 1980). Mengden (1981), however, considers that we need to distinguish two categories of heterochromatinization in relation to sex chromosome differentiation: primary and secondary. With regard to secondary heterochromatinization only one example is known. This is the postulated "progressive heterochromatinization" of the Y-chromosome in the neo XY system of certain orthopterans (Saez, 1963; White, 1973); but the argument supporting this is based on a comparison between largely unrelated species, so that its validity remains to be demonstrated. 
In a previous paper (Fernández-Piqueras et al., 1982) we have described a polytypic species of Tettigonioid (Pycnogaster cucullata) with X0 and neo XY populations. We concluded that the neo XY forms had arisen by a centric fusion between the second largest $M_{2}$ autosome and the $X$ of the $\mathrm{X} 0$ system. This was accompanied by loss in the neo $\mathrm{Y}$ of part of a C-band and the secondary constriction which characterizes the $\mathrm{M}_{2}$, together with the appearance of a new secondary constriction in the distal region of the $\mathrm{X}_{\mathrm{L}}$ limb of the neo $\mathrm{X}$. In this paper, we describe a second neo XY system found in a population of $P$. cucullata from Morcuera which appears to have undergone additional secondary heterochromatinization in both $\mathrm{X}$ and Y chromosomes.

\section{MATERIAL AND METHODS}

Individuals of Pycnogaster cucullata Charp. (Tettigonioidea, Orthoptera) have been studied from natural populations at Gredos (X0;20 individuals), Truchas (neo XY $\mathrm{XY}_{1} ; 0$ individuals), and Morcuera (neo $\mathrm{XY}_{2}$; 20 individuals).

Conventional acetic orcein squash preparations were used together with C-banding preparations (Sumner, 1972).

A silver technique described by Goodpasture and Bloom (1975) and Bloom and Goodpasture (1976) has also been employed though with some modifications. The silver method serves to detect both active NORs (nucleolar organizer regions) (Engel et al., 1977; Hansmann et al., 1978; Schmid et al., 1977; Höfgartner et al., 1979; Buys et al., 1979) and centromeric regions (Buys and Osinga, 1980; Howell and Hsu, 1979). NORs may be distinguished by the fact that the amount of silver precipitate is variable during meiotic prophase, decreasing from zygotene to diakinesis. By constrast, at centromere regions the amount remains unchanged during all stages of meiosis. In Pycnogaster we found that a pretreatment with 2XSSC at $60^{\circ} \mathrm{C}$ for $15 \mathrm{~min}$ allows one to specifically identify centromeric regions.

Air drying and counterstaining in 1 per cent phosphate buffered $(p \mathrm{H} 6$, 8) Giemsa for five min can be used additionally to define the outline of the chromosome.

\section{Results}

\section{(i) XO system Gredos population}

As an addition to our previous findings (Fernández-Piqueras et al., 1982), silver staining has allowed us to determine that there is a NOR region located in the $\mathbf{M}_{2}$ autosome at a procentric site (fig. 1(c)). The secondary constriction is located close to the centromere in the short arm of the $\mathrm{M}_{2}$ (see fig. 3).

\section{(ii) Neo $X Y_{1}$ system Truchas population}

C-banding. We have already shown (Fernández-Piqueras et al., 1982) that the neo $\mathrm{X}$ possesses a prominent procentric $\mathrm{C}$-band with a minor band near the distal tip of the $\mathrm{X}_{\mathrm{L}}$ limb (三 attached autosome for X0 state) (see also here figs. 2(a) and (b)). In some cases, as in fig. 2(a), there appears to 


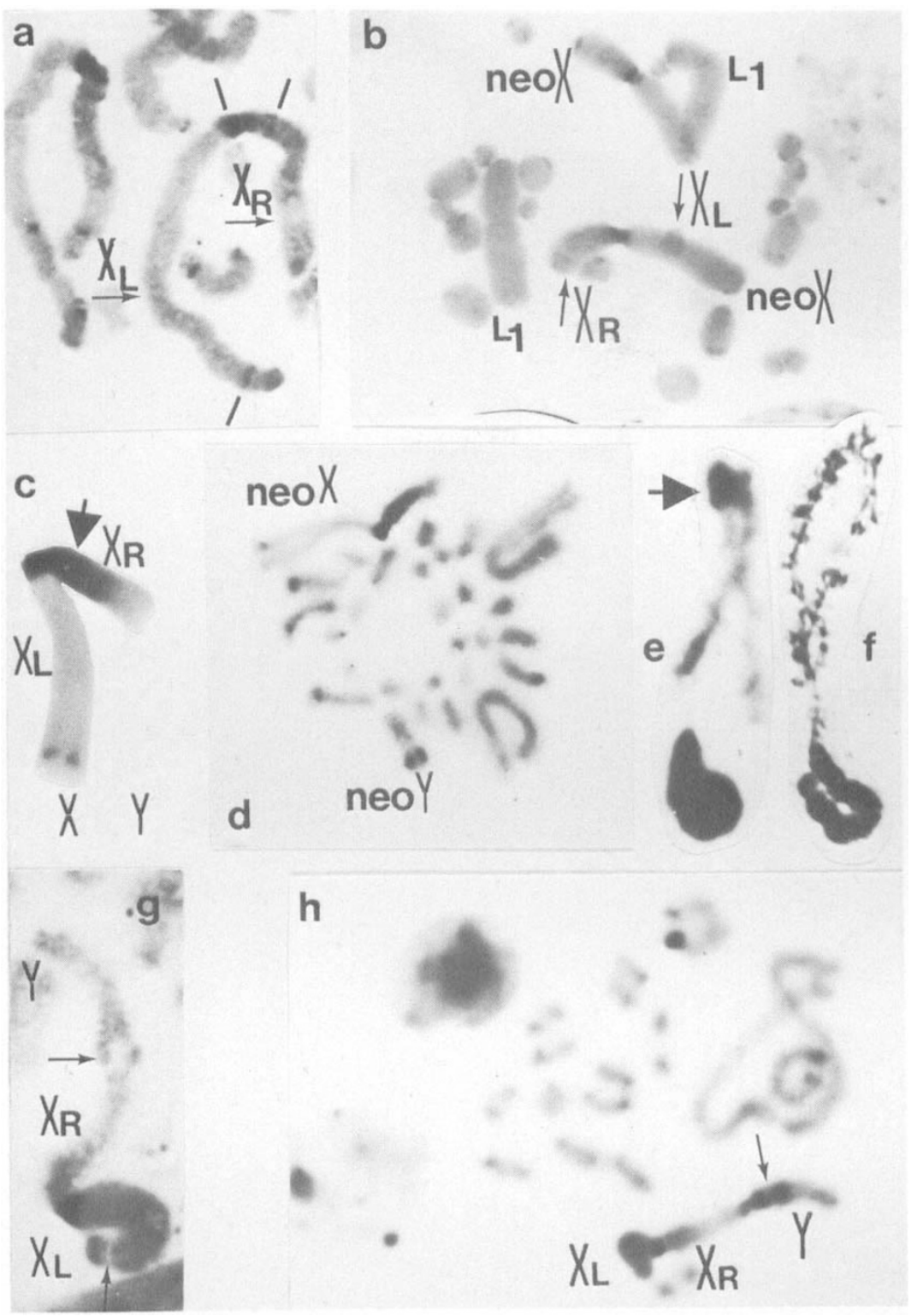

FIG. 2. C-banding and conventional stain. (a) C-banded neo $\mathrm{X}$ chromosome from a female of the neo $\mathrm{XY}_{1}$ system, at mitotic prophase. Bars indicate C-bands. Note a small terminal "grey" C-banding areas in the distal extremities of both $\mathrm{X}_{\mathrm{L}}$ and $\mathrm{X}_{\mathrm{R}}$ arms. (b) C-banded mitotic metaphase from a female of the neo $X_{1}$ system. (c) C-banded neo $X$ and neo $\mathrm{Y}$ at mitotic prometaphase, from a male of the neo $\mathrm{XY}_{2}$ system. Note a "grey" C-block in the proximal half of $X_{R}$ limb (arrow). (d) C-banded mitotic prometaphase from neo $\mathrm{XY}_{2}$. (e) An early prophase sex bivalent from neo $\mathrm{XY}_{2}$, with conventional stain. Note a positive heteropycnotic region on the telomeric region of neo $Y$ (arrow). (f) Idem from neo $X Y_{1}$. Now, the mentioned above heteropycnotic region is absent. (g) C-banded neo $\mathrm{XY}_{1}$ sex bivalent at diplotene. Note subterminal chiasma (arrow), a procentric C-band in the neo $X$ and the distal secondary constriction of the $X_{L}$ arm. (h) C-banded diplotene from neo $\mathrm{XY}_{2}$ system. Note arrow indicates $C$-positive block on the neo $\mathrm{Y}(\mathrm{Y})$. 


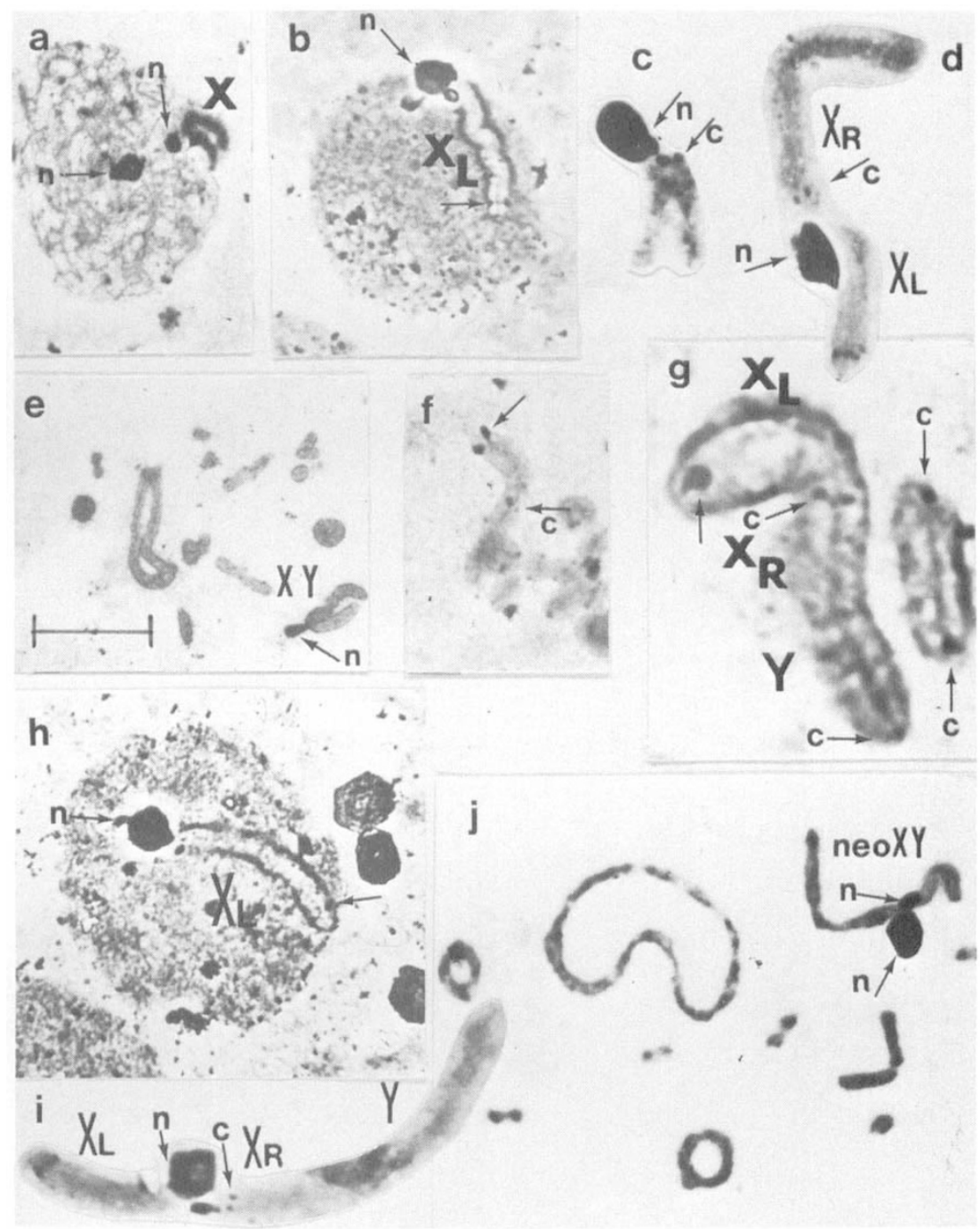

FIG. 1. Ag-staining. $n$, represents nucleolus; $c$, denotes centromeres. (a) Premeiotic nucleus from an $X 0$ individual. (b) Premeiotic nucleus from the neo $X Y_{1}$. The outline of the $X_{L}$ is marked. Arrow indicates two silver spots on the distal secondary constriction of the $X_{L}$. (c) A pachytene $M_{2}$ bivalent from Gredos population. (d) An early pachytene neo $\mathrm{XY}_{1}$ sex bivalent. (e) Diplotene from $\mathrm{XY}_{1}$ state. Note reduced size of $\mathrm{Ag}$-precipitate with regard to that in pachytene and premeiotic stages. (f) Partial metaphase I from $\mathrm{XY}_{1}$. Note centromeres present in all chromosomes. (g) Idem after Giemsa staining. (h) Premeiotic nucleus from the neo $\mathrm{XY}_{2}$. Arrow marks two silver spots on the distal secondary constriction of the $X_{1}$. (i) An early pachytene neo $X Y_{2}$ sex bivalent. (j) Diplotene from $\mathrm{XY}_{2}$ state. 
be a region of differential C-banding in the proximal region of $X_{R}$ which is somewhat reminiscent of the "grey" region that we describe later in the $\mathrm{X}_{\mathrm{R}}$ of the neo $\mathrm{XY}_{2}$ system. Additionally the $\mathrm{X}_{\mathrm{L}}$ limb has an intermediate staining character during meiotic prophase after $\mathrm{C}$-banding treatment, while the $X_{R}$ limb, like the $Y$ does not display any differential staining (fig. 2(g) for $\mathrm{XY}_{1}$ and $2(\mathrm{~h})$ for $\mathrm{XY}_{2}$ ).

Ag-staining. An active NOR is located close to the centromere of the neo $\mathrm{X}$, in the neo $\mathrm{XY}_{1}$ system (figs. $1(\mathrm{~d})$ and (e)). The precise spatial relationship between the centromere and the active NOR can be shown in prophase neo XY bivalents (fig. 1(d) and fig. 3). The Y chromosome of the neo $X Y_{1}$ system does not show any NOR activity.

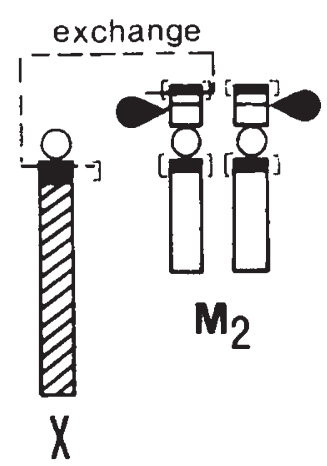

XO STATE

centromere

[ C-banding

C NOR region

- silver dots
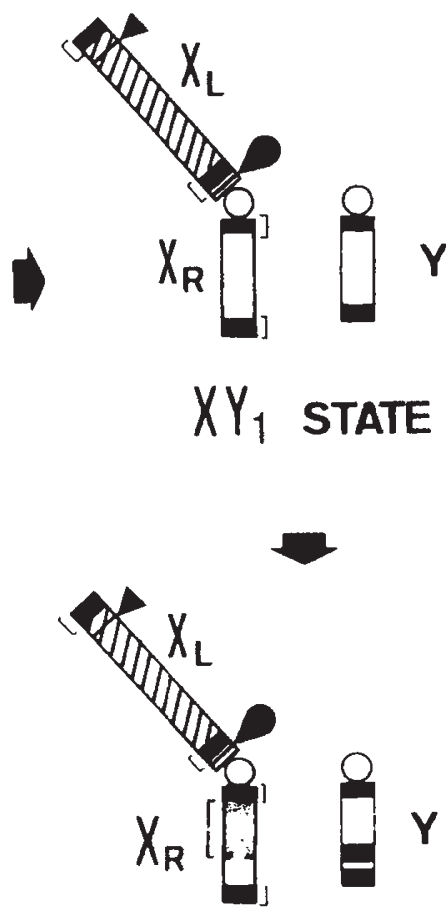

$X Y_{2}$ STATE

FIG. 3. A drawing to summarise the possible origin and evolution of the neo XY systems of this species.

The silver precipitate which is found at the secondary constriction on the distal half of the $X_{L}$ in the neo $X$ is larger than that found at centromeric regions but it remains unchanged during meiosis (figs. 1(a), (f) and (g)), which suggests it may be of centromeric origin.

\section{(iii) $\mathrm{Neo} \mathrm{XY}_{2}$ system Morcuera population}

C-banding. A C-positive block is present at the distal region of the neo $Y_{2}$ but is not present either in its partner $X_{R}$ or in the neo $Y$ of the neo $X Y_{1}$ system. This block consists of two C-bands (fig. 2(h)) but these 
are not always resolvable when either C-banding (fig. 2(a), (c) and (d)) or conventional staining techniques (fig. 2(e)) are used.

A "grey" C-block, which occupies the proximal half of the $X_{R}$ limb of the neo $\mathrm{X}$ in the neo $\mathrm{XY}_{2}$ system, is found during mitotic prophase and prometaphase (figs. 2(c) and (d)). This C-block is not present during all stages of meiosis. As is also the case in the neo $\mathrm{XY}_{1}$ system, the neo $\mathrm{X}$ possesses a procentric $\mathrm{C}$-band and a $\mathrm{C}$-positive secondary constriction towards the distal tip of the long arm. At meiosis, though not at mitosis, the $\mathrm{X}_{\mathrm{L}}$ arm is characterised by intermediate C-staining.

In both neo $X Y$ systems the short $X_{R}$ arm of the neo $X$ is longer than its free partner neo $Y$ (see fig. 2(c) for the neo $X Y_{2}$ and Fernández-Piqueras et al. (1982) for the neo $\left.\mathrm{XY}_{1}\right)$.

Ag-staining. No significant differences are evident between the sex chromosomes of $X Y_{2}$ and $X Y_{1}$ populations after silver staining. Thus, the neo $\mathrm{X}$ of the $\mathrm{XY}_{2}$ system possesses an active NOR close to the centromere, denoted by two silver precipitates (nucleoli) with different sizes present around the proximal secondary constriction during meiotic prophase (fig. 1(j)). The centromere silver dots are located distal to this secondary constriction in the $X_{R}$ arm (fig. 1(i)).

Two silver spots are also present at the distal secondary constriction on the $\mathrm{X}_{\mathrm{L}}$ arm (fig. 1(h)). The neo $\mathrm{Y}$ again does not show any NOR activity (fig. 1(i) and (j)).

We include a drawing (fig. 3) to summarise the origin and evolution of the two neo XY systems in this species.

\section{(iv) Nature of the $X Y$ bivalents}

All of the 300 neo $\mathrm{XY}_{2}$ bivalents examined had a single terminal association between the distal ends of the neo $Y$ and the $X_{R}$ arm of the neo X. Since both these regions are C-banding positive (fig. $2 \mathrm{~h}$ ) it is unlikely that this association represents a terminalised chiasma. Equivalent nonchiasmate associations have been observed in other orthopterans (John and King, 1977 and 1980).

Of $300 \mathrm{XY}_{1}$ bivalents analysed, 198 also showed a terminal association of the distal ends of the $X_{R}$ and the $Y$. In the remainder, however, there was evidence of a subterminal chiasma (fig. $2(\mathrm{~g})$ ). These data suggest a possible difference in the mode of association of the $\mathrm{X}$ and $\mathrm{Y}$ chromosome in the two categories of neo sex systems.

\section{Discussion}

\section{(i) Progressive heterochromatinization}

According to White (1973) fifty species of grasshoppers are characterized by a neo XY mechanism. Of the six such systems that have been described in the Tettigonioidea, those of Yorkiella picta, Polichne parvicauda, Caedicia marginata, and probably, Anabrus simplex, are structurally unmodified. The two other systems show considerable modification from their presumed original state as result of an inversion and/or the development of allocycly in the proximal portion of the neo Y (Hewitt, 1979). Unlike the tendency of the neo $Y$ chromosome to undergo secondary changes associated with its production, the neo $\mathrm{X}$ does not usually give 
evidence of any marked changes. The acrocentric neo X of Dichro plus silveiraguidoi, however, is exceptional in this respect (Diaz and Saez, 1968).

When a comparative study is made between the two neo XY systems of Pycnogaster, two main differences can be observed (fig. 3). First, the development of a constitutive heterochromatic C-block in the distal region of the neo $\mathrm{Y}$ of the neo $X Y_{2}$ which the equivalent neo $\mathrm{Y}$ of the neo $X Y_{1}$ system does not possess. This heterochromatinization process is not accompanied by any size modification of the neo $\mathrm{Y}$, because this is still shorter than the $X_{R}$ as is also the case in the neo $X Y_{1}$ (as it had been shown in a previous paper Fernández-Piqueras et al., 1982). Second, the development of a proximal "grey" C-block of the $X_{R}$ limb of the neo $X$ in the neo $X Y_{2}$ system which is not present in the neo $X$ of the neo $X Y_{1}$ system. This C-block is eupycnotic at all stages of both mitosis and meiosis with conventional procedures, but stains differentially during mitotic prophase and prometaphase after C-banding treatment (fig. 2(c) and (d)).

This "grey" C-block appears to be consequence of a facultative heterochromatinization process, since it displays an intermediate staining and its presence is restricted to the sex chromosomes.

Differences in stain intensity in the sex chromosomes after C-banding have been reported in many cases (Hsu, 1971; Jalal et al., 1974; Stock et al., 1974; Vistorin et al., 1977; Ryttmann et al., 1979) including orthopterans (see for example Cardoso and Dutra, 1979). Whether the intermediate staining observed when sex chromosomes are C-banded is due to differences in DNA composition or to differences in the pattern of chromatin packing is not known.

The heterochromatinization process involving the $X_{R}$ limb is quite different from that present in the $\mathrm{Y}_{2}$ chromosome. The appearance of the C-block in the neo $Y_{2}$ presumably results from a secondary heterochromatinization process that has apparently involved euchromatin transformation of the type described by King (1980) since the $Y_{1}$ and $Y_{2}$ are similar in size.

Unlike the neo XY, system of $P$. cucullata, where a subterminal chiasma can often be demonstrated in the sex bivalent (fig. 2(g)), in the neo $X Y_{2}$ system the two sex chromosomes are invariably associated terminally (fig. $1(\mathrm{j})$ and $2(\mathrm{~h})$ ). It is conceivable that the constitutive heterochromatinization of the neo $\mathrm{Y}_{2}$ may have played a key role in this relationship, since such an event would be expected to lead to a novel mode of association between the $\mathrm{X}$ and $\mathrm{Y}$. There are, however, a number of neo $\mathrm{XY}$ systems known where the $\mathrm{X}$ and the $\mathrm{Y}$ are regularly associated terminally but where there is no distal heterochromatinization of the neo $\mathrm{Y}$. Consequently it is difficult to argue for a causal mechanism based on heterochromatinization without first defining the nature of the terminal association observed in many of the $\mathrm{XY}_{1}$ bivalents of Pycnogaster.

The neo $\mathrm{XY}_{2}$ system we have described here, appears to be the first convincing example of what Saez (1963) described as "progressive heterochromatinization" though with one significant difference. In Saez's original gradient of heterochromatinization hypothesis it was assumed that the $Y$ become heterochromatic at its centric end first and this then spread progressively along the Y. In $P$. cucullata, however, the neo $\mathrm{Y}_{2}$ has become heterochromatic at its distal end and it is the $\mathrm{X}$ which displays proximal heterochromatinization. 


\section{(ii) Ag-staining}

Two other points of interest regarding the evolution of the neo XY sex chromosomes of $P$. cucullata merit comment. First the identification of the centromere and NOR positions on the $M_{2}$ and the neo XY bivalents following $\mathrm{Ag}$-stain demonstrates that the centric fusion between the $\mathrm{M}_{2}$ autosome and the $\mathrm{X}$ of the $\mathrm{X} 0$ system has probably been accompanied by the loss of the centromere region of the $X$ and of the active NOR of the neo $Y$ (figs. 1(d), (f) and (g)). Thus the centromere of the neo $X$ is located on the eupycnotic $X_{R}$ arm, close to the NOR region, occupying the same position it had on the progenitor $\mathrm{M}_{2}$ autosome (compare figs. 1(c) and (d)). On the other hand, while both $\mathrm{M}_{2}$ chromosomes in the X0 state have one active NOR, it has not been possible to demonstrate such activity in the derived neo $\mathrm{Y}$.

The second issue relates to the presence of a secondary constriction on the distal half of the $X_{L}$ limb of the neo $X$ in both neo $X Y$ systems, but which is absent on the free $\mathrm{X}$-chromosome in $\mathrm{X} 0$ state. Since the amount of silver precipitate remains unchanged at all stages of meiosis in this secondary constriction (compare figs. 1(a), (f) and (g)), we believe that this precipitate does not reflect any synthetic activity in that region. The question remains: what does it represent? It might be an atypical centromere (larger than the normal one) or some other structural protein. In the former case, the abnormal centromere could be derived by a pericentric inversion of the $\mathrm{X}$-chromosome during the development of the neo XY system. We consider this interpretation very unlikely though the existence of a centromere in this region would be in the line with the fact that the two arms of the neo $X$ do not always flex at first metaphase. Neocentric activity is certainly not unknown even in the univalent $\mathrm{X}$ of $\mathrm{X} 0$ orthopterans (John, 1976) and such activity could account for the absence of arm flexure.

It is clear that there is no uniform solution applicable to the evolution of all sex chromosome systems. Nevertheless, the neo $\mathrm{XY}_{2}$ system of $P$. cucullata seems to provide some substantiation for regarding "progressive heterochromatinization" as one mechanism involved in the secondary sex chromosome differentiation of orthopterans.

Acknowledgements. We wish to thank Prof. Bernard John for his critical reading of this manuscript and Eugenio Morales Agacino for the taxonomic determination of the material studied by us; without such assistance this paper would not have been written.

\section{REFERENCES}

BLOOM, S. E. AND GOODPASTURE, C. 1976. An improved technique for selective silver staining of nucleolar organizer regions in human chromosomes. Hum. Genet., 34, 199206.

BUYS, C. H. C. M. AND OSINGA, J. 1980. Abundance of protein bound sulfhydryl and disulfide groups at chromosomal nucleolus organizing regions. Chromosoma (Berl.), 77, 1-11.

BUYS, C. H. C. M., OSINGA, J. AND ANDERS, G. J. P. A. 1979. Age-dependence variability of ribosomal RNA-gene activity in man as determined from frequencies of silver staining nucleolus organizing regions on metaphase chromosomes of lymphocytes and fibroblasts. Mech. Age. Dev., 11, 55-75.

CARdoso, H. AND DUTRA, A. 1979. The neo $X$ neo $Y$ sex pair in Acrididae, its structure and association. Chromosoma (Berl.), 70,323-336. 
DIAZ, M. O. AND SAEZ, F. A. 1968. DNA synthesis in the neo X neo Y sex determining system of Dichroplus bergi. Chromosoma (Berl.), 24, 10-16.

ENGEL, W. ZENES, M. T. AND SCHMID, M. 1977. Activation of mouse ribosomal RNA genes at the 2-cell stage. Hum. Gen., 38, 57-63.

FERNANDEZ-PIQUERAS, J., RODRIGUEZ CAMPOS, A., SENTIS CASTANO, C., AND WANDOSELL JURADO, F. 1982. Pycnogaster cucullata (Carp.) a polytypic species of Tettigonioidea with X0 and neo XY sex chromosomes. Heredity, 48 (1), 147-150.

GOODPASTURE, C. AND BLOOM, S. E. 1975. Visualization of nucleolus organizer regions in mammalian chromosomes using silver stain. Chromosoma (Berl.), 53, 37-50.

GROSSMAN, A. I., SHORT, R. B. AND CAIN, G. C. 1981. Karyotype evolution and sex chromosome differentiation in Schistosomes (Trematoda, Schistosomatidae). Chromosoma (Berl.), 84, 413-430.

HANSMANN, I., GEBAUER, J., BIHL, L. AND GRIMM, T. 1978. On set of nucleolus organizer activity in early mouse embryogenesis and evidence for its regulation. Exp. Cell. Res., $144,263-268$.

HEWITT, G. M. 1979. Grasshoppers and Crickets. Animal Cytogenetics. Vol. 3, Insecta 1, Orthoptera. Gebrüder Borntraeger, Berlin-Stuttgart.

HÖFGARTNER, F. J., SCHMID, M., KRONE, W., ZENZES, M. T. AND ENGEL, W. 1979. Patterns of activity of nucleolus organizers during spermatogenesis in mammals as analysed by silver staining. Chromosoma (Berl.), 71, 197-216.

HOWELL, W. M. AND HSU, T. C. 1979. Chromosome core structure revealed by silver staining. Chromosoma (Berl.), 73, 61-66.

HSU, T. C. 1971. Heterochromatin pattern in metaphase chromosomes of Drosophila melanogaster. J. Hered., 62, 285-287.

JALAL, S. M., CLARK, R. W., HSU, T. C. AND PATHAK, S. 1974. Cytological differentiation of constitutive heterochromatin. Chromosoma (Berl.), 48, 391-403.

JOHN, B. 1976. Myths and mechanisms of meiosis. Chromosoma (Berl.), 54, 295-325.

JOHN, B. AND KING, M. 1977. Heterochromatin variation in Cryptobothrus chrysophorus I. Chromosome differentiation in natural populations. Chromosoma (Berl.), 64, 219-236.

JOHN, B. AND KING, M. 1980. Heterochromatin variation in Cryptobothrus chrysophorus III. Synthetic hybrids. Chromosoma (Berl.), 78, 165-186.

KING, M. 1980. C-banding studies on the Australian hylid frogs: secondary constriction structure and the concept of euchromatin transformation. Chromosoma (Berl.), 80, $191-217$.

MENGDEN, G. A. 1981. Linear differentiation of the C-band pattern of the $\mathrm{W}$ chromosomes in snakes and birds. Chromosoma (Berl.), 83, 275-287.

RAY-CHAUDHURI, S. P., SINGH, L. AND SHARMA, T. 1971. Evolution of sex chromosomes and formation of W chromatin in snakes. Chromosoma (Berl.), 33, 239-251.

RYTTMAN, H., TGELSTROM, H. AND JANSSON, H. 1979. G and C-banding in four related Larus species (Aves). Hereditas (Lund), 91, 143-148.

SAEZ, F. A. 1963. Gradient of the heterochromatinization in the evolution of the sexual system neo X neo Y. Port. Acta Biol. A., 7, 111-138.

SCHMID, M., HÖFGARTNER, F. J., ZENZES, M. T. AND ENGEL, W. 1977. Evidence for post-meiotic expression of ribosomal RNA genes during male gametogenesis. Hum. Genet., 38, 279-284.

SCHMID, M., OLERT, J. AND KLETT, C. 1979. Chromosome banding in Amphibia. III. Sex chromosomes in Triturus. Chromosoma (Berl.), 71, 29-55.

SINGH, L., PURDOM, I. F. AND JONES, R. W. 1980. Sex chromosome associated satellite DNA: evolution and conservation. Chromosoma (Berl.), 79, 137-157.

STOCK, A. D., ARRIGHI, F. E. AND STEFOS, K. 1974. Chromosome homology in birds; banding patterns of the chromosomes of the domestic chicken ring-necked dove and domestic pidgeon. Cytogenet. Cell Genet., 13, 419-428.

SUMNER, A. T. 1972. A simple technique for demonstrating centromeric heterochromatin. Ex. Cell Res., 75, 304-306.

VISTORIN, G., GAMPERL, R. AND ROSENKRANZ, w. 1977. Studies on sex chromosomes of four hamster species: Cricetus cricetus, Cricetulus griseus, Mesocricetus auratus and Phodopus sungorus. Cytogenet. Cell Genet., 18, 24-32.

WHITE, M. J. D. 1973. Animal cytology and evolution. 3rd edit. Cambridge University Press. 